\title{
Genome-Wide Analysis of $P R-1$ Genes From Piper Nigrum Reveals Critical Role in Defense Response During Phytophthora Capsici Infection
}

Divya Kattupalli

Rajiv Gandhi Centre for Biotechnology

Asha Srinivasan

Rajiv Gandhi Centre for Biotechnology

Soniya E V ( $\sim$ evsoniya@rgcb.res.in )

Rajiv Gandhi Centre for Biotechnology

\section{Research Article}

Keywords: Foot rot, Black pepper, Promoter, CAP domain, Plant immunity, Cis-regulatory element, Biotic stress

Posted Date: December 30th, 2020

DOI: https://doi.org/10.21203/rs.3.rs-130603/v1

License: (c) (7) This work is licensed under a Creative Commons Attribution 4.0 International License. Read Full License 


\section{Abstract}

Background: Black pepper is a prominent spice which is an indispensable ingredient in culinary and traditional medicine. Phytophthora capsici, the causative agent of foot rot disease causes drastic constraint in black pepper cultivation and productivity. To counterattack various biotic and abiotic stresses plants employ a broad array of mechanisms one such includes the accumulation of pathogenesis-related $(P R)$ proteins. Several studies have reported the role of $P R-1$ proteins in triggering the plant defenses during plant-oomycete interaction.

Results: Through the genome-wide survey, eleven $P R-1$ genes that belongs to a CAP superfamily protein with Caveolin-Binding Motif (CBM) and CAP-derived peptide (CAPE) were identified from P. nigrum. Despite the critical functional domains, $P n P R 1$ homologs differ in their signal peptide motifs, and core amino acid sequence composition in the functional protein domains. The GO, biological function analysis reveals their role in defense responses and response to biotic stimulus whereas the KEGG functional annotation predicted their function in the plant-pathogen interactions. Furthermore, transcriptome-assisted FPKM analysis revealed $P n P R-1$ genes mapped to $P$. nigrum - $P$. capsici interaction pathway. The differentially expressed pathogen-responsive $P R$ - 1 gene was validated through qRT-PCR. Subsequent analysis revealed the structural details, phylogenetic relationships, conserved sequence motifs and critical cis-regulatory elements of PnPR-1 genes.

Conclusion: This is the first genome-wide study that identified the role of $P R-1$ genes during $P$. nigrum - $P$. capsici interactions. The detailed in silico experimental analysis revealed the vital role of $P n P R-1$ genes in regulating the first layer of defense towards $P$. capsici infection in Panniyur-1 plants.

\section{Introduction}

Plant immunity involves multiple layers of defense responses. The first layer of defense is triggered by the detection of microbe-associated molecular patterns (MAMPs) through plant-, pathogen- or pattern-recognition receptors (PRRs), which activates PAMP / pathogen / pattern - triggered immunity (PTI) [1]. Plants defense machinery was forced to evolve continuously to combat a wide range of challenges project through harmful pathogens and pests. These challenges activate an array of induced mechanisms such as hypersensitive response (HR) which involves a series of events including the production of reactive oxygen species (ROS), synthesis of antimicrobial molecules and pathogenesis related (PR) proteins. PR proteins induces programmed cell death which further inhibits the spread of infection contribute to systemic acquired resistance (SAR) [2-5]. In the second layer of defense, pathogens suppresses the PTI by secreting the effector proteins which are later recognized by plant resistance $(\mathrm{R})$ proteins leading to effector-triggered immunity [1].

Salicylic acid and the subsequent activation of PR genes are necessary for the establishment of SAR in the distant regions of infections [6]. Arabidopsis mutants deficient in Nonexpressor of Pathogenesis Related 1 (NPR1) protein, a key SAR regulator showed less PR gene expression which in turn increased the susceptibility to pathogens [7]. Various plant species overexpressing Arabidopsis NPR1 in other plant species displayed enhanced disease resistance to a variety of pathogens [8-10]. Till date 17 families of PR proteins have been classified and characterized [11]. PR proteins are generally functionally diverse proteins that are inducible during pathogen attack, and are regulated by the signalling compounds such as abscisic acid (ABA), ethylene (ET), jasmonic acid (JA) and salicylic acid (SA) $[12,13]$. 
$P R-1 a$, the first member of $P R-1$ family was identified in Nicotiana tabacum plants infected with Tobacco Mosaic Virus. $P R-1$ proteins belong to the group of most abundantly produced proteins during plant defense responses, and they are ubiquitous across plant species [14]. $P R-1$ proteins are involved in the cell wall thickening and thereby prevents the spread of the pathogens in the apoplast (Wang et al., 2013). The induction of certain members of $P R-1$ proteins upon pathogen infection have been studied in many plants [15]. Apart from the biotic stresses, the role of $P R-1$ in abiotic stresses [16-18] were also reported. The upregulation, abundance and the localization in the apoplast make them a potential candidate for broad antimicrobial activity [19]. An enhanced tolerance to fungi [20], oomycetes [21] and bacterial infections [22] were demonstrated with overexpression of $P R$ 1 in the transgenic plants. Apart from its in role in various biotic and abiotic stresses $P R-1$ and $P R$ - 1 like proteins have reported to be involved in plant flowering [23] and seed development [24] respectively.

$P R-1$ proteins are widely reported across the plant kingdom. These are the members of cysteine-rich secretory protein, antigen 5, and pathogenesis-related-1 (CAP) protein superfamily. Stress signalling peptide such as CAPE1 (Cap-derived peptide 1) are embedded within $P R-1$ proteins. CAPE-1 peptide that comprises of last 11 amino acids from the C-terminus of the $P R-1$ protein was reported from tomato plants subjected to wounding and methyl jasmonate treatment [25]. A caveolin-binding motif (CBM) in the CAP region which is involved in sterol binding is responsible for antimicrobial activity against oomycetes, including Phytophthora and Pythium species which require exogenous sterols for basic metabolism. Plants with enhanced $P R-1$ expression are particularly well protected against oomycete pathogens [26]. The in-depth structural and biochemical analysis $P R-1$ proteins can provide more insights into their function during defense signalling in crop plants.

In the world of spices, black pepper (Piper nigrum L., family Piperaceae) is considered as 'The King of spices' due to its pungent constituent. It is a major additive in many ayurvedic medicinal preparations. The major hindrance in the black pepper production is the destructive foot rot or quick wilt disease caused by an oomycete Phytophthora capsici [27, 28]. In this work, to extend our knowledge on the defense mechanisms underlying PR function in $P$. nigrum, we carried out a comprehensive genome-wide analysis and validation of $P R-1$ genes from $P$. nigrum. Transcriptome assisted analysis and expression profiling revealed the differential expression of $P R-1$ genes during $P$. capsici infection in P. nigrum variety Panniyur-1.

\section{Results}

\section{Genome wide identification and analysis of P. nigrum PR-1 genes}

Eleven potential $P n P R-1$ gene candidates were obtained from the genome-wide analysis in comparison to the $A$. thaliana PR genes. The number of exons harboring in each PnPR-1 gene is one, expect in Pn2.357 which has two exons. The length of PnPR-1 proteins ranged from 127 to 357 amino acid residues with molecular weight ranging from 14.38 to $38.49 \mathrm{kDa}$. The theoretical isoelectric point ( $\mathrm{pl}$ ) data categorized PnPR-1 proteins into acidic (Pn21.1032 and Pn36.35) and others as basic in nature (Table 1). Furthermore, the signal peptide regions and cleavage sites were also identified in all the PnPR-1 protein sequences except Pn11_1637 and Pn31.171 (Table 2). 
Table 1

Sequence characteristics and physio-chemical properties of the PR-1 proteins in Piper nigrum.

\begin{tabular}{|c|c|c|c|c|c|c|c|c|c|}
\hline $\begin{array}{l}\text { Genome } \\
\text { CDS Id }\end{array}$ & $\begin{array}{l}\text { Genome } \\
\text { Scaffold } \\
\text { No. }\end{array}$ & $\begin{array}{l}\text { Exon } \\
\text { No. }\end{array}$ & Start & Stop & Strand & $\begin{array}{l}\text { Protein } \\
\text { length } \\
\text { (AA) }\end{array}$ & $\begin{array}{l}\text { Molecular } \\
\text { weight } \\
\text { (kDa) }\end{array}$ & Theol & etical pl \\
\hline Pn2.460 & Pn23 & 1 & 1315655 & 1316146 & - & 155 & 17.09488 & 4.82 & \multirow[t]{2}{*}{ Acidic } \\
\hline Pn2.459 & Pn23 & 1 & 1320928 & 1321419 & - & 192 & 21.91361 & 5.8 & \\
\hline Pn2.433 & Pn23 & 1 & 1493563 & 1494054 & - & 168 & 18.35074 & 7.58 & \multirow[t]{9}{*}{ Basic } \\
\hline Pn2.357 & Pn23 & 2 & 2226595 & 2229193 & - & 127 & 14.37881 & 7.62 & \\
\hline Pn2.340 & Pn23 & 1 & 2402850 & 2403341 & - & 185 & 19.80756 & 9.1 & \\
\hline Pn21.1032 & Pn3 & 1 & 3202437 & 3202904 & + & 163 & 17.85002 & 9.15 & \\
\hline Pn31.171 & Pn15 & 1 & 26854949 & 26855332 & - & 163 & 17.87807 & 9.3 & \\
\hline Pn36.35 & Pn25 & 1 & 5754975 & 5755553 & - & 163 & 17.88005 & 9.3 & \\
\hline Pn11.1637 & Pn4 & 1 & 33322945 & 33323502 & - & 176 & 19.63337 & 9.37 & \\
\hline Pn8.549 & Pn8 & 1 & 25779763 & 25780269 & - & 163 & 17.96421 & 9.44 & \\
\hline Pn14.1312 & Pn14 & 1 & 5932334 & 5932864 & + & 357 & 38.49596 & 11.3 & \\
\hline
\end{tabular}

Table 2

Signal peptide region detected from the PnPR-1 proteins

\begin{tabular}{|lllllll|}
\hline Genome Id & $\begin{array}{l}\text { Cleavage site } \\
\text { position }\end{array}$ & $\begin{array}{l}\text { Sequence } \\
\text { position }\end{array}$ & Probability & $\begin{array}{l}\text { Protein } \\
\text { type }\end{array}$ & $\begin{array}{l}\text { Signal Peptide } \\
\text { (Sec/SPI) }\end{array}$ & Other \\
\hline Pn21.1032 & 16 and 17 & CNA-QN & 0.9541 & Likelihood & 0.9981 & 0.0019 \\
\hline Pn2.340 & 24 and 25 & AQA-QN & 0.8849 & Likelihood & 0.9984 & 0.0016 \\
\hline Pn2.357 & 24 and 25 & AQA-QN & 0.8898 & Likelihood & 0.9986 & 0.0014 \\
\hline Pn2.433 & 24 and 25 & AQA-QN & 0.8892 & Likelihood & 0.9987 & 0.0013 \\
\hline Pn2.459 & 24 and 25 & AQA-QN & 0.8898 & Likelihood & 0.9986 & 0.0014 \\
\hline Pn2.460 & 24 and 25 & AQA-QN & 0.8892 & Likelihood & 0.9987 & 0.0013 \\
\hline Pn36.35 & 29 and 30 & ASS-SP & 0.6292 & Likelihood & 0.9813 & 0.0187 \\
\hline Pn14.1312 & 31 and 32 & TNA-AL & 0.4399 & Likelihood & 0.7324 & 0.2676 \\
\hline Pn8.549 & 30 and 31 & TLA-QN & 0.3454 & Likelihood & 0.5106 & 0.4894 \\
\hline Pn31.171 & - & - & - & Likelihood & 0.0029 & 0.9971 \\
\hline Pn11.1637 & - & - & - & Likelihood & 0.2691 & 0.7309 \\
\hline
\end{tabular}

All the eleven PnPR-1 protein sequences were identified as potential members of CAP superfamily with CAP domain structure indicated in green line, along with caveolin-binding motif (CBM; marked in yellow) and CAPderived peptide (CAPE; marked in blue) motif (Fig. 1). Comparison of PnPR-1 sequences with various other monocot and dicot plants showed distinct CAP domain with CBM and CAPE (Fig. S1). The signal peptide regions 
(Pink colored boxes) of PnPR-1, were also represented in Fig. 1 and the cleavage site predicted was indicated by the arrowhead.

\section{Sequence conservation of PnPR-1 genes}

The nucleotide and protein sequence conservation of all the PnPR-1 candidates were checked using Mega 7. The subsequent phylogenetic analysis of all eleven $P R-1$ nucleotide and protein sequences through the maximumlikelihood method with 1000 bootstraps (Fig. S2A \& S2B) revealed two main clusters leaving one outnumbered group (Pn21.1032). A total of ten conserved motifs were identified using MEME server. Motif 1 and 2 were conserved in all the deduced $P R-1$ proteins whereas motif 3 and motif 6 are conserved in all $P n P R-1$ proteins except in Pn31.171 and Pn2.357 respectively. Motif 8, motif 9 and motif 10 were preserved in Pn36.35 along with Pn14.1312, Pn31.171, Pn1.1637 respectively (Fig. 2).

\section{GO and KEGG pathway analysis of PnPR-1 genes}

Gene ontology (GO) analysis carried out using PANNZER2 online server yielded five biological processes, four molecular functions and four cellular components. Based on the $\mathrm{GO}$ enrichment analysis, predicted PR proteins have their roles in all the three classes. Most of the $P R-1$ genes have their role in defense responses and response to biotic stimulus in biological function. In terms of molecular function, it has protein kinase activity, adenyl nucleotide and purine ribonucleoside binding activities. Cellular component showed its role in extracellular region (Table 3). Kegg pathways analysis categorized its role in Environmental Information Processing- Signal transduction pathways like MAPK signaling pathway - plant (04016), Plant hormone signal transduction (04075) and Plant-pathogen interaction (04626).

Table 3

The gene ontology (GO) term distribution of $P n P R-1$ proteins

\begin{tabular}{|lll|}
\hline GO Id & GO Domain & Function Description \\
\hline G0:0006952 & Biological process & Defense response \\
\hline G0:0009607 & Biological process & Response to biotic stimulus \\
\hline G0:0048544 & Biological process & Recognition of pollen \\
\hline G0:0010274 & Biological process & Hydrotropism \\
\hline G0:0004672 & Molecular function & Protein kinase activity \\
\hline G0:0030554 & Molecular function & Adenyl nucleotide binding \\
\hline G0:0035639 & Molecular function & Purine ribonucleoside triphosphate binding \\
\hline G0:0032555 & Molecular function & Purine ribonucleotide binding \\
\hline G0:0005576 & Cellular component & Extracellular region \\
\hline G0:0016020 & Cellular component & Membrane \\
\hline G0:0031224 & Cellular component & Intrinsic component of membrane \\
\hline
\end{tabular}


A varied percentage of a-helices (15.6-36.88\%), extended strand (10.64-23.78\%), beta turn (3.65-5.88\%) and random coil (37.42-67.79\%) were found in the Pn-PR1 proteins (Fig. S3). 3D-strucures of the Pn-PR1 genes were generated using Phyre2online server and pocket structures were predicted (Fig. 3). The distinct structural variations were detected among the $P n P R-1$ proteins. At the same time, proportion of disordered regions of $P R-1$ proteins ranged from $4.5-28.1 \%$.

\section{Cis-regulatory elements of PnPR-1 genes}

The cis-elements were found to be distributed over the $2.0 \mathrm{~kb}$ upstream promoter region of PnPR-1 genes (Fig. 4). The length of these cis-elements varied from 9 to $42 \mathrm{bp}$ in Softberry database whereas 4 to 24 bp in NewPlace database. Among the ten PR-1 gene loci, the typical TGA binding site LS7, WBSI, G-box and C- motif were found in the promoter regions of Pn36.35. Meanwhile, GT motif and Zc2A/T-2 were found in the Pn2.340, Pn2.433 and Pn2.460. The hormone signalling elements such as ABI4 and GCC-box were present in Pn2.459 and Pn2.340, Pn2.433 respectively. The stress responsive MYB was also detected in the promoter regions of PnPR1 genes such as Pn21.1032, Pn31.171 and Pn36.35. Among 154 cis-elements detected from New Place database, CAAT box (CAAT), E-box (CANNTG), DOFCOREZM (AAAG) regions were found to be widely distributed across the PnPR-1 promoter regions (Fig. S4).

\section{Expression of PR-1 genes during P. capsici infection in P. nigrum}

The assembled transcriptome of RNA-seq data from control and $P$. capsici infected $P$. nigrum plants revealed 60437 transcripts (Communicated). From the assembled data seven transcripts of $P n P R-1$ genes were mapped to the $P$. capsici - $P$. nigrum interaction pathway. The transcripts length ranges from 391-1015bps. Differential expression of these transcripts was assessed from their corresponding FPKM values among control leaf (CL), infected leaf (IL) and infected root (IR). CL10113.C1/2 and Unigene17664 (Fig. 5A-C) that respectively mapped to the Pn23 and Pn8 scaffolds were highly expressed (4.57 to 5.7-fold) in the infected leaf during pathogen infection. Meanwhile Unigene11116, Unigene15555, Unigene26912 and Unigene693 were moderately expressed in IL (Fig. 5D-G). All the transcripts, except Unigene693 were downregulated drastically (2 to 7-fold) in IR compared to IL (Fig. 5 main figure).

\section{RT-qPCR validation of PnPR-1 genes}

The expression of $P n P R-1$ gene was further validated using RT-qPCR from $P$. capsici infected $P$. nigrum plants. In agreement with the transcriptome results, a high upregulation of PnPR-1 gene CL10113.C2 was detected from the infected leaf. Furthermore, the temporal expression at $6 \mathrm{hrs}, 12 \mathrm{hrs}$ and $24 \mathrm{hrs}$ of $P$. capsici infection revealed sequential increase in expression of PnPR-1 genes from 6 to 12 hours of infection (Fig. 6A). A drastic upregulation of PnPR-1 expression was observed in the leaves at 24hours after infection compared to the mock control plant (ML). The trypan blue staining has enabled us to discrimination between intact-viable and damaged regions on leaves. Viable cells were colorless whereas infected tissue was stained in blue (Fig. 6B).

\section{Discussion}

PR proteins are defense-related signaling molecules induced by phytopathogens which play a vital role in resisting the entry of invading pathogen. PR proteins have been classified into many families based on their function, molecular weight, amino acid sequence and other properties [29]. In tobacco PR proteins were initially 
classified as five major classes (PR1, PR2, PR3, PR4 and PR5) [30, 31]. However, later studies in tobacco and tomato have grouped them into 11 families [3]. The members belonging to PR family can be either acidic or basic in nature. Basic PR proteins are located intracellularly in the vacuole regions and are constitutively expressed to some extent and are also induced by stress signals, whereas acidic types were produced extracellularly and only triggered by specific stress signals [32]. In our current study, both acidic and basic $P R$ - 1 proteins which have critical role during P. nigrum: Phytophthora interactions were identified.

KEGG orthology analysis revealed $P$. nigrum $P R-1$ genes mapped to the plant-pathogen interaction (04626), MAPK signaling pathway (04016) and Plant hormone signal transduction (04075). PR-1 family mainly possess antifungal and anti-oomycetes activities [29]. Overexpression of PR1 or like proteins in various plants leads to enhanced disease resistance to a wide variety of pathogens [15] especially the oomycetes [21, 33]. The antioomycete properties of PR-1 proteins such as P14c and PR-1 were demonstrated against the sterol auxotroph pathogen Phytophthora brassicae[26].

As previously reported $[19,26]$, $P$. nigrum $P R-1$ proteins possess the established features such as the CAP tetrad, CBM involved in Sterol binding [34] and CAPE involved in plant immune signalling [25]. The property of $P R-1$ family of proteins to bind sterols contributes towards their antimicrobial activity towards Phytophthora species, a major plant pathogen belonging to sterol auxotroph [26]. The CAPE 1 peptide has the consensus motif PxGNxxxxxPY, that is conserved between the monocots and dicots. Highly conserved and distinct similarity in domains of PR1 protein structure was observed in PnPR-1 proteins which might account to a general strategy in responding to various biotic stresses as reported in other studies [35]. The role of CAPE-1 peptide in the defense signalling was demonstrated from the previous study on Pseudomonas syringae pv tomato (Pst) strain DC3000 interaction in tomato. A diverse set of defense-related genes were induced in the tomato plants pretreated with CAPE-1 peptide. Furthermore, a non-canonical pathway other than PAMP triggered immunity (PTI) signalling was suggested for the CAPE-1 mediated defense responses, as CAPE-1 did not induce the WRKY TRANSCRIPTION FACTOR53 (WRKY53) [25]. The pathogen effectors ToxA and Tox3 proteins from Parastagonospora nodorum were found to interact with wheat $P R-1$ proteins $[36,37]$.

Promoters are the regulators of gene at the transcriptional level [38]. Various computational methods are thoroughly being used for the identification of different cis-elements in the promoter region that are responsible for the regulation of the genes [39]. A range of cis-elements were predicted from $P$. nigrum $P R-1$ genes which were likely related to the regulation of plant growth, development and response to various stresses. A high frequency of CAAT box (CAAT), E-box (CANNTG), DOFCOREZM (AAAG) regions were found in the PnPR1 promoter regions. A high occurrence of CAAT box was previously reported in the A. thaliana PR proteins [40]. As previously reported, hormone regulating sequence motifs [40] such as LS7, GCC-box, ABA responsive elements (ABREs; also termed as G-box) [41] and ABI4 were detected from the upstream of certain PnPR1 genes. LS7 which contains a TGA binding site is reported as the key activator of $P R-1$ expression, NPR1 [42]. Ethylene-responsive factor binds to GCC-box (ethylene-responsive element) and responses to various biotic and abiotic factors in Arabidopsis [43]. ABA-INSENSITIVE-4 (ABI4) transcription factor was reported to be involved in ascorbate-dependent plant growth [44]. AC elements in the promoter region of Leucoanthocyanidin reductase gene in Proanthocyanidins pathway promoter harbors binding site for MYB2 Myb-like transcription factors [45]. Subsequently WER-binding site (WBSI) and NonaLS are also reported in PnPR1 promoter region. WBSI was detected in the CAPRICE (CPC) promoter of Arabidopsis, WEREWOLF (WER) is a MYB protein and gene transcription activator during the specification of epidermal cell fates [46]. NonaLS (Nona like sequence-GATCGGACG) is the positive cis-acting element of histone 
wheat, tomato, and Arabidopsis [47]. Induction of both biotic and abiotic responsive Cis-acting regulatory elements in $P n P R-1$ indicates that these reported $P R-1$ genes from $P$. nigrum may play a key role in regulating resistance against $P$. capsici and other abiotic stresses.

Even though $P R-1$ proteins belong to the group of abundant proteins expressed in plant pathogen interaction, all the members of the family were not uniformly upregulated [19]. Likewise, transcriptome assisted analysis revealed high upregulation of two basic $P R-1$ genes ( $\mathrm{pl}>7.35$ ) such as CL10113.C1/2 and Unigene17664 in $P$. nigrum upon $P$. capsici infection. As $P R-1$ genes belong to the group of multigene families, they differ widely in their properties.

In addition to the host defense response during pathogen infection, $P R-1$ proteins were also reported to have role in abiotic stress stimuli $[17,48-50]$. Studies show the rise in $P R-1$ expression levels both transcriptionally and translationally upon pathogen infections [51,52]. Same pattern of expression was observed in our study where the increased transcript levels were observed in CL10113.Contig1_All, CL10113.Contig2_All, Unigene17664_All. The qRT-PCR expression studies of CL10113.Contig2_All also proved the same. The trypan blue staining clearly shows the necrotic region as the defense responses of the host plant to the pathogen and its further effect to inhibit the growth of pathogen to the surrounding regions. Till date only few studies were carried out on role of $P R$ proteins in $P$. nigrum or related Piperaceae species. The activity of PR protein chitinase, $\beta-1,3$-glucanase and their related enzymes in $P$. capsici infected $P$. nigrum were reported by [53-55]. The present study contributes a significant advancement in the understanding of the molecular function of $P R-1$ proteins in black pepper. $P n P R-1$ genes are found to have key role in the early defense such as PTI towards $P$. capsici infection in Panniyur-1 plants. It may be possible that the key genes in the subsequent effector triggered immunity act as critical players of defense response in Panniyur-1 plants. Therefore, future studies on identification of the potential $P$. capsici effectors coupled with $P R-1$ functional studies will ascertain the in-depth mechanisms of defense signal amplification and anti-oomycete properties of this enigmatic proteins in black pepper.

\section{Conclusion}

The genome-wide survey identified eleven $P$. nigrum $P R-1$ gene homologs mapped to seven distinct genome scaffolds. A subsequent transcriptome analysis of $P$. capsici infected black pepper plants showed the expression of $P R-1$ genes from all the mapped loci. Meanwhile a significantly different expression was detected for CL10113.C2 mapped to the P. nigrum scaffold 23. Detailed in silico analysis revealed cis-regulatory elements such as phytohormone responsive, transcription activators etc. in the promoter regions. The structural analysis revealed similar binding pockets in the predicted 3D structures of all PnPR1 proteins, except Pn31.171. The differential expression of certain PnPR-1 homologs revealed their crucial role during early defense response in $P$. capsici - P. nigrum interaction. Further in-depth functional studies on PnPR-1 genes, promoter cis-regulatory elements and the pathogen-specific effectors can provide us the exact molecular mechanism of the susceptibility /tolerance of black pepper cultivars to Phytophthora infection, which in turn contribute towards the novel disease protection strategies in P. nigrum plants.

\section{Methods}

\section{Identification and analysis of $P R$-1 genes from $P$. nigrum genome}


PR-1 genes of Arabidopsisthaliana were downloaded from TAIR (The Arabidopsis Information Resource) database (https://www.arabidopsis.org/index.jsp) and did tblastn search against the $P$. nigrum genome assemblies [56]. The coding sequences were translated and aligned by multiple sequence alignment using BioEdit Sequence Alignment Editor [57]. The domain structure prediction was carried out using NCBI- Conserved Domain Database (CDD) [58].

The molecular weight and pl of PnPR-1 proteins were estimated by Expasy ProtParam tool (https://web.expasy.org/protparam/). The potential signal peptide regions and the cleavage sites were also predicted using Signal-P 5.0 server (http://www.cbs.dtu.dk/cgi-bin). Subsequently the conserved motifs of PnPR-1 proteins were predicted using MEME (Multiple Em for Motif Elicitation) (http://meme-suite.org/tools/meme) [59].

\section{GO \& KEGG analysis}

Gene ontology (GO) was classified into biological processes, cellular components, and molecular function. PnPR1 genes were analyzed for their role in GO using PANNZER2 web server (http://ekhidna2.biocenter.helsinki.fi/sanspanz/) [60]. KEGG (Kyoto Encyclopedia of Genes and Genomes) tool, BlastKOALA (KEGG Orthology and Links Annotation) server (https://www.kegg.jp/blastkoala/) [61] was used for the individual characterization of gene functions.

\section{Secondary and tertiary structure prediction}

Secondary structure prediction of $P n P R-1$ proteins was predicted using Self-Optimized Prediction Method with Alignment (SOPMA) server (https://npsa-prabi.ibcp.fr/cgi-bin/npsa_automat.pl?page=/NPSA/npsa_sopma.html) [62]. Predicted 3D structures were build using Protein Homology/analogY Recognition Engine v2 (Phyre2) server (http://www.sbg.bio.ic.ac.uk/ phyre2/html/page.cgi?id=index) [63]. The CASTp (Computed Atlas of Surface Topography of proteins) tool (http://sts.bioe.uic.edu/castp/calculation.html) was used to predict the active site pockets and topology of PnPR-1 proteins structures [64].

\section{Role of PnPR-1 in P. capsici infected $P$. nigrum}

Control P. nigrum (uninfected) leaf transcriptome data (SRA050094), P. capsici infected P. nigrum leaf (SRX853366) and root transcriptome data (SRX856639) were reanalyzed and the final assembled data was used for the expression studies. PnPR-1 sequences curated from the P. nigrum genome [56] were mapped to the transcriptome assembly files. The obtained transcripts differential regulation checked using FPKM (Fragments Per Kilobase of transcript per Million mapped reads) values.

\section{Plant-pathogen infections, staining and RT-qPCR}

Virulent, pure cultures of $P$. capsici and root cuttings of $P$. nigrum cultivar panniyur1 were procured from College of Agriculture, Vellayani. 24hr old $P$. capsici culture was infected to the fully expanded second leaf of $P$. nigrum plant. The treatment of mock infection was done with plain PDA discs. To check the temporal expression of $P n P R-1$, the infected leaf samples were collected at $6 \mathrm{hr}, 12 \mathrm{hr}$ and $24 \mathrm{hr}$ after infection. Mock infected plats were used as a control. To detect and visualize the tissue damage in the leaf region after pathogen infection, trypan blue staining was performed [65]. Three biological replicates were used for all the studies. 
RNA was isolated from the collected leaf samples using mirVana miRNA isolation kit (Invitrogen, Cat No: AM1560) according to the manufacturer's instructions. The quality and the quantity of RNA samples were checked by Colibri Microvolume Spectrometer. RNA was reverse transcribed into cDNA using High-Capacity cDNA synthesis Kit (Applied Biosystems, Cat No: 4374966). RT-qPCR was carried out using Applied Biosystems7900 HT sequence detection system (ABI) using SYBR Green qPCR Master Mix (ABI). Each qPCR reaction was conducted in $10 \mu \mathrm{L}$ volume containing $1 \mu \mathrm{L}$ of diluted cDNA $(10 \mathrm{ng} / \mu \mathrm{L}), 5 \mu \mathrm{L}$ of SYBR green and $5 \mathrm{pmol}$ of forward (5'CCCACAAGACTTCCTCAAC3') and reverse (5'CGTAATTCCGTGCGTAGG3') primers with the following conditions: 40 cycles of $95{ }^{\circ} \mathrm{C}$ for $15 \mathrm{~s}$ DNA denaturation, annealing at $60{ }^{\circ} \mathrm{C}$ for $15 \mathrm{~s}$ and elongation at $72{ }^{\circ} \mathrm{C}$ for 30 s. $5.8 S$ RNA was used as an endogenous control [28]. The relative quantification was analyzed by comparative CT method using the formula 2- $\Delta \Delta \mathrm{CT}$ and standard deviation was represented as the error bar [66].

\section{Prediction of cis-acting regulatory elements in Promoter regions}

The genomic sequences of $2.0 \mathrm{kbp}$ upstream of the CoDing Sequence (CDS) region of each $P n P R-1$ gene were extracted from $P$. nigrum genome assembly. Promoter regions of each $P R 1$ genes were scanned for the presence of functional motifs using Softberry (http://www.softberry.com/berry.phtml? topic=case_study_plants\&no_menu=on) and New Place (https://www.dna.affrc.go.jp/PLACE/?action=newplace) [67].

\section{Declarations}

\section{Ethics approval and consent to participate}

Not Applicable

\section{Consent for publication}

Not Applicable

\section{Availability of data and materials}

Control P. nigrum (uninfected) leaf transcriptome data, $P$. capsici infected $P$. nigrum leaf and root transcriptome data can be accessed from NCBI SRA accession numbers SRA050094, SRX853366 \& SRX856639 respectively.

\section{Competing interests}

The authors declare no competing interests.

\section{Funding}

This work was supported by Department of Biotechnology (DBT), India. Grant Number (). The funders have no role in study design, data collection and analysis, decision to publish or preparation of the manuscript.

\section{Author contributions}

K.D, A.S. and E.V.S conceived the research plans and designed the experiments. D.K. and A.S. performed the experiments, analyzed the data and wrote the article, E.V.S. made critical revisions. All the authors read and approved the manuscript. 
K.D. acknowledge Department of Biotechnology (DBT) - JRF \& SRF and Commonwealth Scholarship Commission (CSC) for Split-site scholarship. The A.S. greatly acknowledge fellowship from Council of Scientific and Industrial Research (CSIR) - JRF \& SRF (A.S.). E.V.S. acknowledges financial support from DBT, New Delhi.

\section{References}

1. Dangl JL, Jones JDG. defence responses to infection. 2001;411 June.

2. Van Baarlen P, Van Belkum A, Summerbell RC, Crous PW, Thomma BPHJ. Molecular mechanisms of pathogenicity: How do pathogenic microorganisms develop cross-kingdom host jumps? FEMS Microbiology Reviews. 2007.

3. van Loon LC, Pierpoint WS, Boller T, Conejero V. Recommendations for naming plant pathogenesis-related proteins. Plant Mol Biol Report. 1994.

4. Van Loon LC, Van Strien EA. The families of pathogenesis-related proteins, their activities, and comparative analysis of PR-1 type proteins. Physiol Mol Plant Pathol. 1999.

5. Chassot C, Nawrath C, Métraux JP. Cuticular defects lead to full immunity to a major plant pathogen. Plant J. 2007.

6. Boccardo NA, Segretin ME, Hernandez I, Mirkin FG, Chacón O, Lopez Y, et al. Expression of pathogenesisrelated proteins in transplastomic tobacco plants confers resistance to filamentous pathogens under field trials. Sci Rep. 2019.

7. Cao H, Bowling SA, Gordon AS, Dong X. Characterization of an Arabidopsis Mutant That Is Nonresponsive to Inducers of Systemic Acquired Resistance. Plant Cell. 1994.

8. Le Henanff G, Farine S, Kieffer-Mazet F, Miclot AS, Heitz T, Mestre P, et al. Vitis vinifera VvNPR1.1 is the functional ortholog of AtNPR1 and its overexpression in grapevine triggers constitutive activation of PR genes and enhanced resistance to powdery mildew. Planta. 2011.

9. Molla KA, Karmakar S, Chanda PK, Sarkar SN, Datta SK, Datta K. Tissue-specific expression of Arabidopsis NPR1 gene in rice for sheath blight resistance without compromising phenotypic cost. Plant Sci. 2016.

10. Malnoy M, Jin Q, Borejsza-Wysocka EE, He SY, Aldwinckle HS. Overexpression of the apple MpNPR1 gene confers increased disease resistance in Malus x domestica. Mol Plant-Microbe Interact. 2007.

11. Christensen AB, Cho BHO, Næsby M, Gregersen PL, Brandt J, Madriz-Ordeñana K, et al. The molecular characterization of two barley proteins establishes the novel PR-17 family of pathogenesis-related proteins. Mol Plant Pathol. 2002.

12. Loake G, Grant M. Salicylic acid in plant defence-the players and protagonists. Current Opinion in Plant Biology. 2007.

13. van Loon LC, Rep M, Pieterse CMJ. Significance of Inducible Defense-related Proteins in Infected Plants. Annu Rev Phytopathol. 2006.

14. Van Loon LC, Van Kammen A. Polyacrylamide disc electrophoresis of the soluble leaf proteins from Nicotiana tabacum var. "Samsun" and "Samsun NN". II. Changes in protein constitution after infection with tobacco mosaic virus. Virology. 1970. 
15. Mitsuhara I, Iwai T, Seo S, Yanagawa Y, Kawahigasi H, Hirose S, et al. Characteristic expression of twelve rice PR1 family genes in response to pathogen infection, wounding, and defense-related signal compounds (121/180). Mol Genet Genomics. 2008.

16. Akbudak MA, Yildiz S, Filiz E. Pathogenesis related protein-1 (PR-1) genes in tomato (Solanum lycopersicum L.): Bioinformatics analyses and expression profiles in response to drought stress. Genomics. 2020.

17. Seo PJ, Lee AK, Xiang F, Park CM. Molecular and functional profiling of Arabidopsis pathogenesis-related genes: Insights into their roles in salt response of seed germination. Plant Cell Physiol. 2008.

18. Hon Wai-Ching, Griffith M, Mlynarz A, Kwok YC, Yang DS. Antifreeze proteins in winter rye are similar to pathogenesis-related proteins. Plant Physiol. 1995.

19. Breen S, Williams SJ, Outram M, Kobe B, Solomon PS. Emerging Insights into the Functions of PathogenesisRelated Protein 1. Trends in Plant Science. 2017.

20. Kiba A, Nishihara M, Nakatsuka T, Yamamura S. Pathogenesis-related protein 1 homologue is an antifungal protein in Wasabia japonica leaves and confers resistance to Botrytis cinerea in transgenic tobacco. Plant Biotechnol. 2007.

21. Sarowar S, Young JK, Eui NK, Ki DK, Byung KH, Islam R, et al. Overexpression of a pepper basic pathogenesisrelated protein 1 gene in tobacco plants enhances resistance to heavy metal and pathogen stresses. Plant Cell Rep. 2005.

22. Shin SH, Pak JH, Kim MJ, Kim HJ, Oh JS, Choi HK, et al. An acidic PATHOGENESIS-RELATED1 gene of Oryza grandiglumis is involved in disease resistance response against bacterial infection. Plant Pathology Journal. 2014.

23. Lotan T, Ori N, Fluhr R. Pathogenesis-related proteins are developmentally regulated in tobacco flowers. Plant Cell. 1989.

24. Cooper B, Clarke JD, Budworth P, Kreps J, Hutchison D, Park S, et al. A network of rice genes associated with stress response and seed development. Proc Natl Acad Sci U S A. 2003.

25. Chen YL, Lee CY, Cheng KT, Chang WH, Huang RN, Nam HG, et al. Quantitative peptidomics study reveals that a wound-induced peptide from PR-1 regulates immune signaling in tomato. Plant Cell. 2014.

26. Gamir J, Darwiche R, van't Hof P, Choudhary V, Stumpe M, Schneiter R, et al. The sterol-binding activity of PATHOGENESIS-RELATED PROTEIN 1 reveals the mode of action of an antimicrobial protein. Plant J. 2017.

27. Vandana V V., Suseela Bhai R, Azeez S. Biochemical defense responses of black pepper (Piper nigrum L.) lines to Phytophthora capsici. Physiol Mol Plant Pathol. 2014;88:18-27. doi:10.1016/j.pmpp.2014.06.003.

28. Asha S, Soniya E V. Transfer RNA Derived Small RNAs Targeting Defense Responsive Genes Are Induced during Phytophthora capsici Infection in Black Pepper (Piper nigrum L.). Front Plant Sci. 2016;7 June:1-16. doi:10.3389/fpls.2016.00767.

29. AGRIOS GN. HOW PLANTS DEFEND THEMSELVES AGAINST PATHOGENS. In: Plant Pathology. 2005.

30. van Loon LC, Gerritsen YAM, Ritter CE. Identification, purification, and characterization of pathogenesisrelated proteins from virus-infected Samsun NN tobacco leaves. Plant Mol Biol. 1987.

31. Bol JF, Linthorst HJM, Cornelissen BJC. Plant Pathogenesis-Related Proteins Induced by Virus Infection. Annu Rev Phytopathol. 1990.

32. Memelink J, Linthorst HJM, Schilperoort RA, Hoge JHC. Tobacco genes encoding acidic and basic isoforms of pathogenesis-related proteins display different expression patterns. Plant Mol Biol. 1990. 
33. Alexander D, Goodman RM, Gut-Rella M, Glascock C, Weymann K, Friedrich L, et al. Increased tolerance to two oomycete pathogens in transgenic tobacco expressing pathogenesis-related protein 1a. Proc Natl Acad Sci U S A. 1993.

34. Choudhary V, Darwiche R, Gfeller D, Zoete V, Michielin O, Schneiter R. The caveolin-binding motif of the pathogen-related yeast protein Pry1, a member of the CAP protein superfamily, is required for in vivo export of cholesteryl acetate. J Lipid Res. 2014.

35. Lincoln JE, Sanchez JP, Zumstein K, Gilchrist DG. Plant and animal PR1 family members inhibit programmed cell death and suppress bacterial pathogens in plant tissues. Mol Plant Pathol. 2018.

36. Breen S, Williams SJ, Winterberg B, Kobe B, Solomon PS. Wheat PR-1 proteins are targeted by necrotrophic pathogen effector proteins. Plant J. 2016.

37. Lu S, Faris JD, Sherwood R, Friesen TL, Edwards MC. A dimeric PR-1-type pathogenesis-related protein interacts with ToxA and potentially mediates ToxA-induced necrosis in sensitive wheat. Mol Plant Pathol. 2014.

38. Danino YM, Even D, Ideses D, Juven-Gershon T. The core promoter: At the heart of gene expression. Biochimica et Biophysica Acta - Gene Regulatory Mechanisms. 2015.

39. Kaur G, Pati PK. Analysis of cis-acting regulatory elements of Respiratory burst oxidase homolog (Rboh) gene families in Arabidopsis and rice provides clues for their diverse functions. Comput Biol Chem. 2016.

40. Kaur A, Pati PK, Pati AM, Nagpal AK. In-silico analysis of cis-acting regulatory elements of pathogenesisrelated proteins of Arabidopsis thaliana and Oryza sativa. PLoS One. 2017.

41. Ross C, Shen QJ. Computational prediction and experimental verification of HVA1-like abscisic acid responsive promoters in rice (Oryza sativa). Plant Mol Biol. 2006.

42. Kesarwani M, Yoo J, Dong X. Genetic interactions of TGA transcription factors in the regulation of pathogenesis-related genes and disease resistance in Arabidopsis. Plant Physiol. 2007.

43. Fujimoto SY, Ohta M, Usui A, Shinshi H, Ohme-Takagi M. Arabidopsis ethylene-responsive element binding factors act as transcriptional activators or repressors of GCC box-mediated gene expression. Plant Cell. 2000.

44. Foyer $\mathrm{CH}$, Kerchev PI, Hancock RD. The ABA-INSENSITIVE-4 (ABI4) transcription factor links redox, hormone and sugar signaling pathways. Plant Signal Behav. 2012.

45. Akagi T, Ikegami A, Yonemori K. DkMyb2 wound-induced transcription factor of persimmon (Diospyros kaki Thunb.), contributes to proanthocyanidin regulation. Planta. 2010.

46. Ryu KH, Kang YH, Park YH, Hwang I, Schiefelbein J, Lee MM. The WEREWOLF MYB protein directly regulates CAPRICE transcription during cell fate specification in the Arabidopsis root epidermis. Development. 2005.

47. Taoka Kl, Ohtsubo N, Fujimoto Y, Mikami K, Meshi T, Iwabuchi M. The modular structure and function of the wheat $\mathrm{H} 1$ promoter with S phase-specific activity. Plant Cell Physiol. 1998.

48. Seo PJ, Kim MJ, Park JY, Kim SY, Jeon J, Lee YH, et al. Cold activation of a plasma membrane-tethered NAC transcription factor induces a pathogen resistance response in Arabidopsis. Plant J. 2010.

49. Kothari KS, Dansana PK, Giri J, Tyagi AK. Rice stress associated protein 1 (OsSAP1) interacts with aminotransferase (OsAMTR1) and pathogenesis-related 1a protein (OsSCP) and regulates abiotic stress responses. Front Plant Sci. 2016.

50. Liu WX, Zhang FC, Zhang WZ, Song LF, Wu WH, Chen YF. Arabidopsis Di19 functions as a transcription factor and modulates PR1, PR2, and PR5 expression in response to drought stress. Mol Plant. 2013. 
51. Ali S, Mir ZA, Bhat JA, Tyagi A, Chandrashekar N, Yadav P, et al. Isolation and characterization of systemic acquired resistance marker gene PR1 and its promoter from Brassica juncea. 3 Biotech. 2018.

52. Tunsagool P, Jutidamrongphan W, Phaonakrop N, Jaresitthikunchai J, Roytrakul S, Leelasuphakul W. Insights into stress responses in mandarins triggered by Bacillus subtilis cyclic lipopeptides and exogenous plant hormones upon Penicillium digitatum infection. Plant Cell Rep. 2019.

53. Nazeem PA, Achuthan CR, Babu TD, Parab G V., Girija D, Keshavachandran R, et al. Expression of pathogenesis related proteins in black pepper (Piper nigrum L.) in relation to Phytophthora foot rot disease. $J$ Trop Agric. 2008.

54. Trang Anh T, Bao Linh T, Vu Phong N, Lan Thanh Bien T, Thi Nha Tram T, Dinh Don L. Expression of Proteins Related to Phytophthora capsici Tolerance in Black Pepper (Piper nigrum L.). Int J Agric Innov Res. 2018.

55. P VKI, K JG, Anandaraj M. Real - Time Quantitative Rt-Pcr of Some Defense Inoculated With Phytophthora Capsici. 2016;6:69-78.

56. Hu L, Xu Z, Wang M, Fan R, Yuan D, Wu B, et al. The chromosome-scale reference genome of black pepper provides insight into piperine biosynthesis. Nat Commun. 2019.

57. Hall TA. BIOEDIT: a user-friendly biological sequence alignment editor and analysis program for Windows 95/98/ NT. Nucleic Acids Symp Ser. 1999.

58. Marchler-Bauer A, Derbyshire MK, Gonzales NR, Lu S, Chitsaz F, Geer LY, et al. CDD: NCBI's conserved domain database. Nucleic Acids Res. 2015.

59. Bailey TL, Johnson J, Grant CE, Noble WS. The MEME Suite. Nucleic Acids Res. 2015.

60. Törönen P, Medlar A, Holm L. PANNZER2: A rapid functional annotation web server. Nucleic Acids Res. 2018.

61. Kanehisa M, Sato Y, Morishima K. BlastKOALA and GhostKOALA: KEGG Tools for Functional Characterization of Genome and Metagenome Sequences. J Mol Biol. 2016.

62. Geourjon C, Deléage G. Sopma: Significant improvements in protein secondary structure prediction by consensus prediction from multiple alignments. Bioinformatics. 1995.

63. Kelley LA, Mezulis S, Yates CM, Wass MN, Sternberg MJE. The Phyre2 web portal for protein modeling, prediction and analysis. Nat Protoc. 2015.

64. Dundas J, Ouyang Z, Tseng J, Binkowski A, Turpaz Y, Liang J. CASTp: Computed atlas of surface topography of proteins with structural and topographical mapping of functionally annotated residues. Nucleic Acids Res. 2006.

65. Fernández-Bautista N, Domínguez-Núñez J, Moreno MM, Berrocal-Lobo M. Plant Tissue Trypan Blue Staining During Phytopathogen Infection. BIO-PROTOCOL. 2016.

66. Livak KJ, Schmittgen TD. Analysis of relative gene expression data using real-time quantitative PCR and the 2(-Delta Delta C(T)) Method. Methods. 2001.

67. Higo K, Ugawa Y, Iwamoto M, Korenaga T. Plant cis-acting regulatory DNA elements (PLACE) database: 1999. Nucleic Acids Research. 1999.

\section{Figures}


Pn14.1312

Pn31.171

Pn2. 460

Pn2. 459

Pn2. 433

Pn2. 340

Pn 36.35

Pn21. 1032

Pn11.1637

Pn8. 549
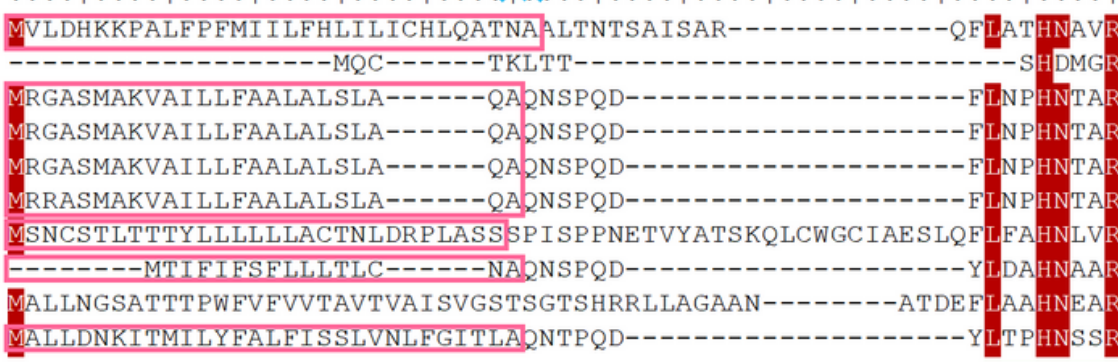

ATHNAVRSAVGMPELRW

. $\mid \ldots$.

. $\ldots . . \ldots$.

$$
110 \quad 120 \quad 130
$$

140

$150 \quad 160$
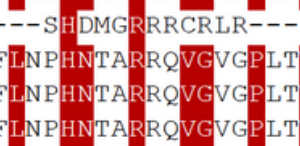

QVAMRYAEWYY

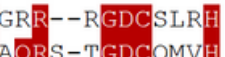

\section{$.1 \ldots$}

$120=130$

$160 \quad 170$

180

Pn14.1312

Pn31.171

Pn2. 460

Pn2. 459

Pn2. 433

Pn2. 340

Pn 36.35

Pn21.1032

Pn11.1637

Pn8. 549
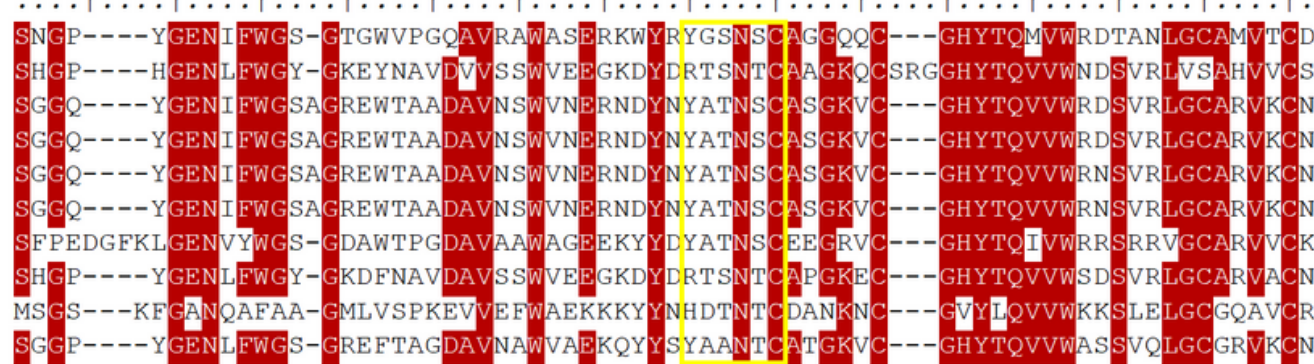

..1
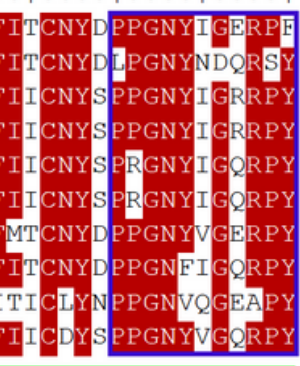

\section{Figure 1}

Multiple sequence alignments of the PnPR-1 protein sequences. Pink colored rectangle boxes indicate the signal peptide regions, cleavage site predicted were indicated in blue arrowheads. The green line indicates CAP domain structure of $150 \mathrm{bp}$. Yellow and blue color rectangles show the caveolin-binding motif (CBM) and CAP-derived peptide (CAPE) respectively. 


\section{Name}

Pn14.1312

Pn31.171

Pn2.460

Pn2.459

Pn2.433

Pn2.357

Pn2.340

Pn36.35

Pn21.1032

Pn11.1637

Pn8.549 $p$-value Motif Locations

\subsection{7e-99}

$6.55 e-78$

7.13e-161

1.63e-160

$1.73 e-159$

$1.52 \mathrm{e}-138$
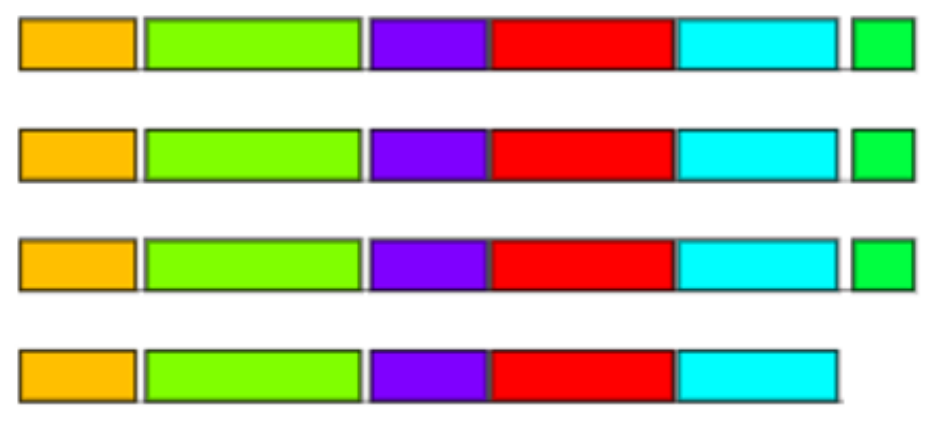

$1.42 \mathrm{e}-155$

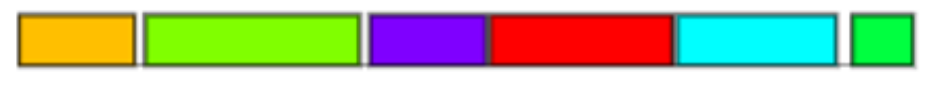

$1.83 e-91$

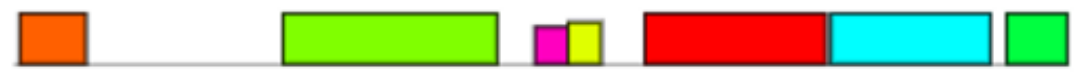

4.89e-118
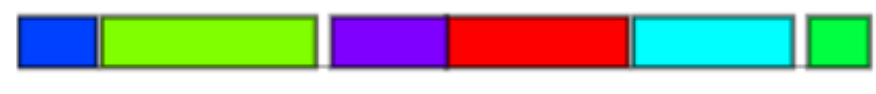

$1.46 e-57$

$2.93 e-116$
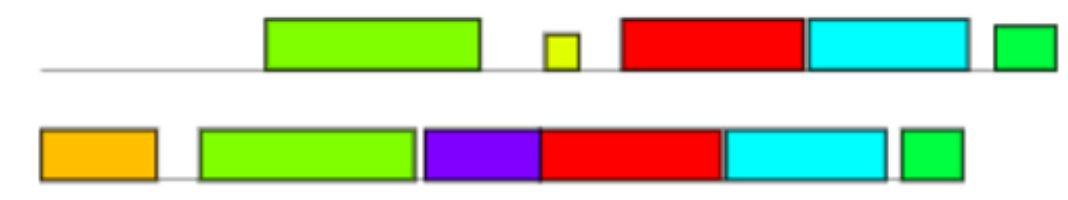

\section{Motif Symbo \\ 1. $\square$ \\ 3. \\ 4. \\ 5. \\ 6. \\ 8. \\ 9. \\ 10.}

\section{Motif Consensus}

GREWTAADAVNSWVNERKDYNYATNSCASGKVC

HYTQVVWRDSVRIGCARVKCNNGGVFIIC

AQNSPQDFLNPHNTARRQVGVGPLTWDPTVAAYAQNYAN

IGDCRIVHSGGQYGENI FWGS

MRGASMAKVAILLFAALALSL

PRGNYIGZRPY

FPEFIFLFHLJLJC

LDHKFP

MNCSKLTTSHDI

EDGFKF

Figure 2

Conserved motifs identified from Piper nigrum PR-1 protein homologs. A total of 10 conserved motifs were identified. Each color represents different motifs with consensus sequences. 


\section{Pn14.1312}

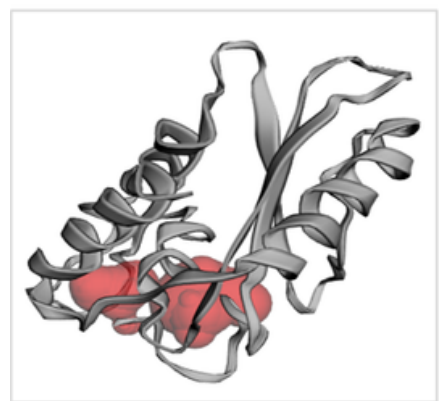

\section{Pn2.357}

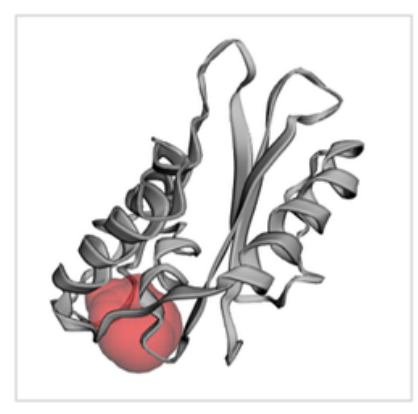

Pn31.171

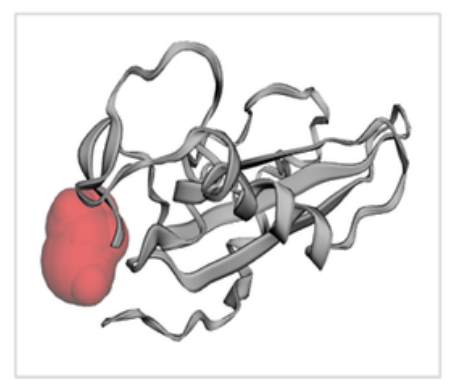

Pn11.1637

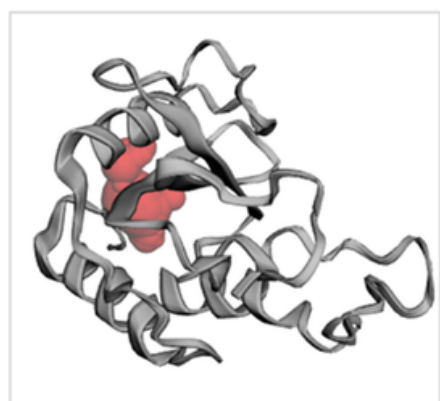

Pn2.340

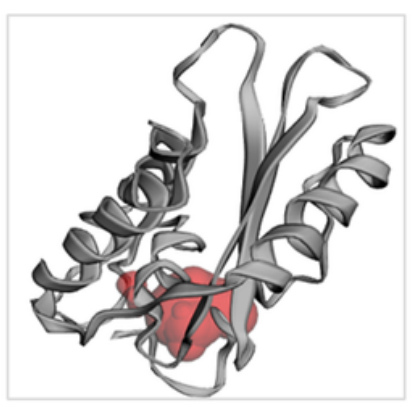

Pn2.433

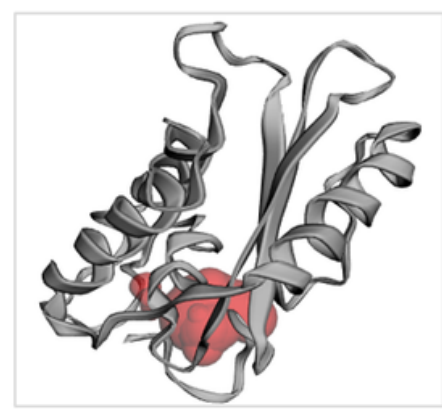

Pn2.460

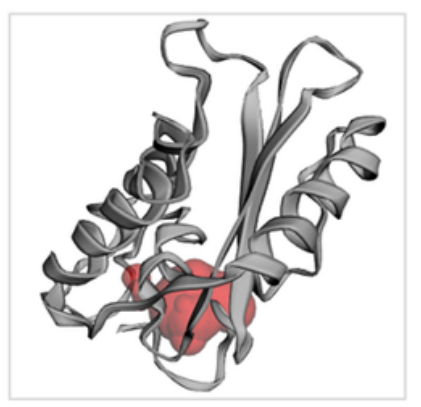

Pn2.459

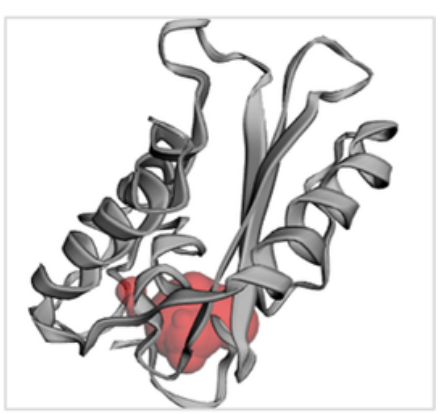

Pn8.549

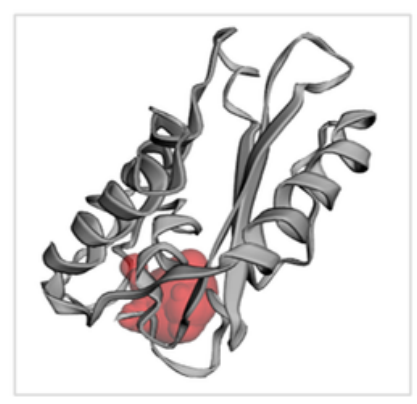

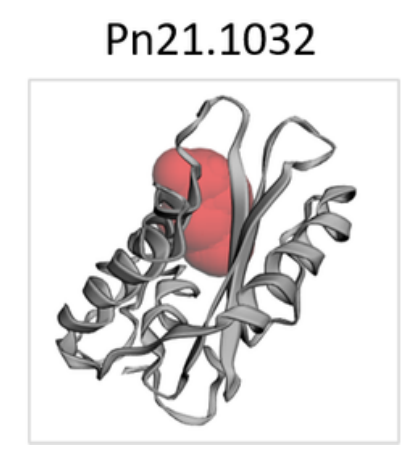

Pn36.35

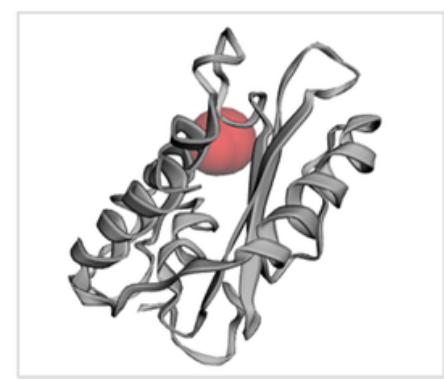

Figure 3

The predicted 3D structures of PnPR-1 proteins generated using the Phyre2 server and binding pockets identified by the CASTp 3.0 server (red color). 


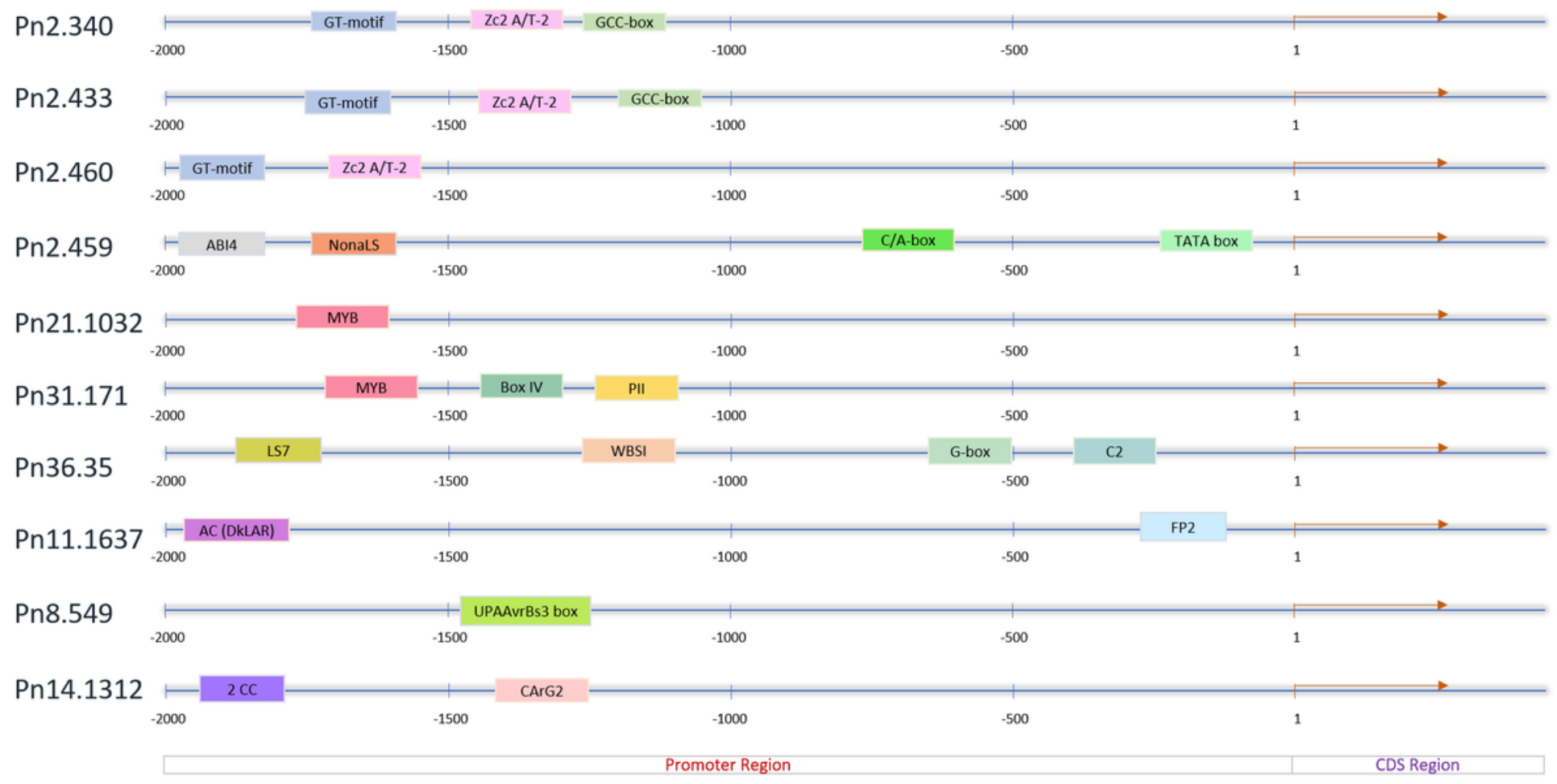

\section{Figure 4}

Analysis of cis-acting elements of the PnRR-1 promoter regions ( $2 \mathrm{~kb}$ upstream from the CDS region) using the Softberry Software. Each element was represented with different colors.

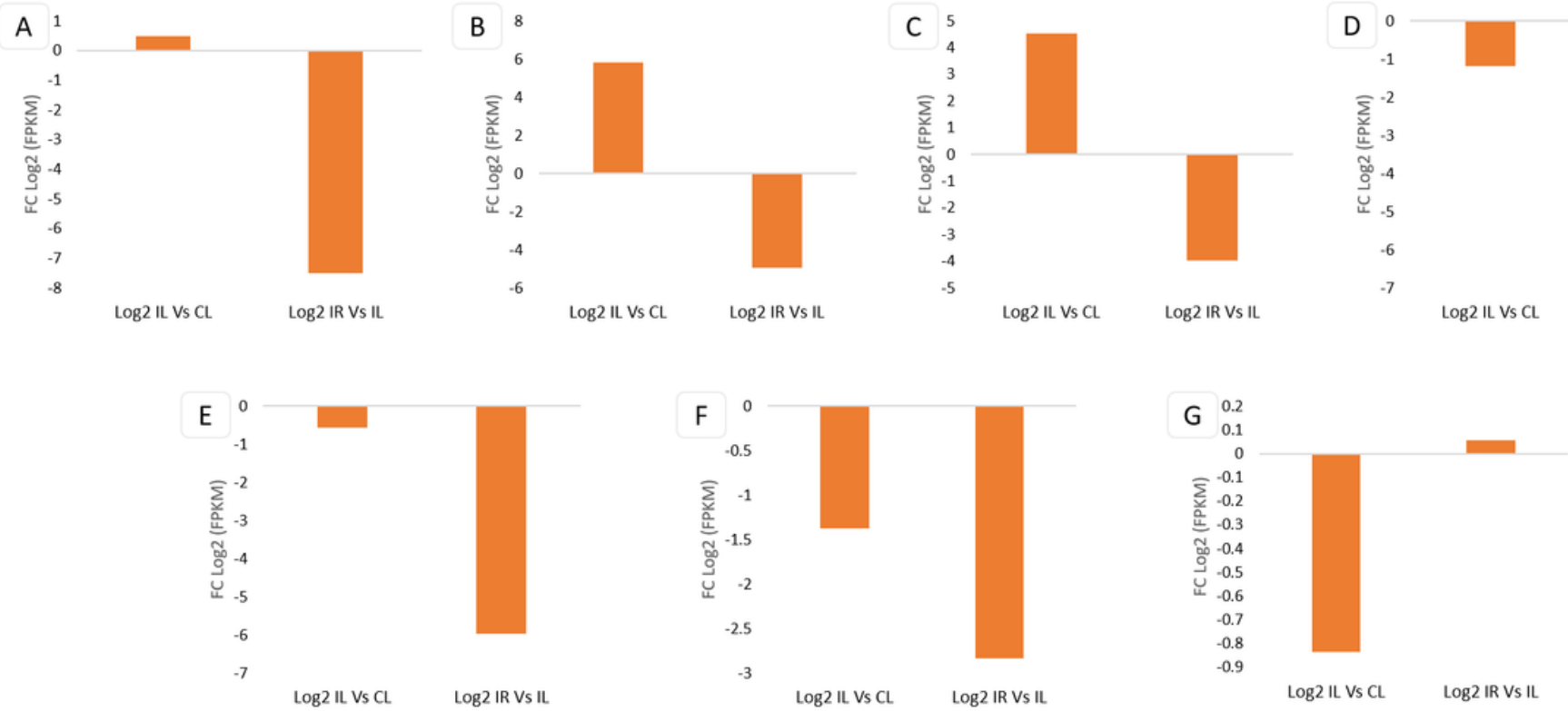

Figure 5

Expression analysis of the PnPR-1 transcripts in Phytophthora capsici infected P. nigrum using FPKM (Fragments Per Kilobase of transcript per Million mapped reads) values. (A) CL10113.C1 (B) CL10113.C2 (C) Unigene17664 (D) Unigene11116 (E) Unigene15555 (F) Unigene26912 (G) Unigene693. Positive and negative numbers on the $X$ axis indicate fold changes Log2 gene expression levels. 
A

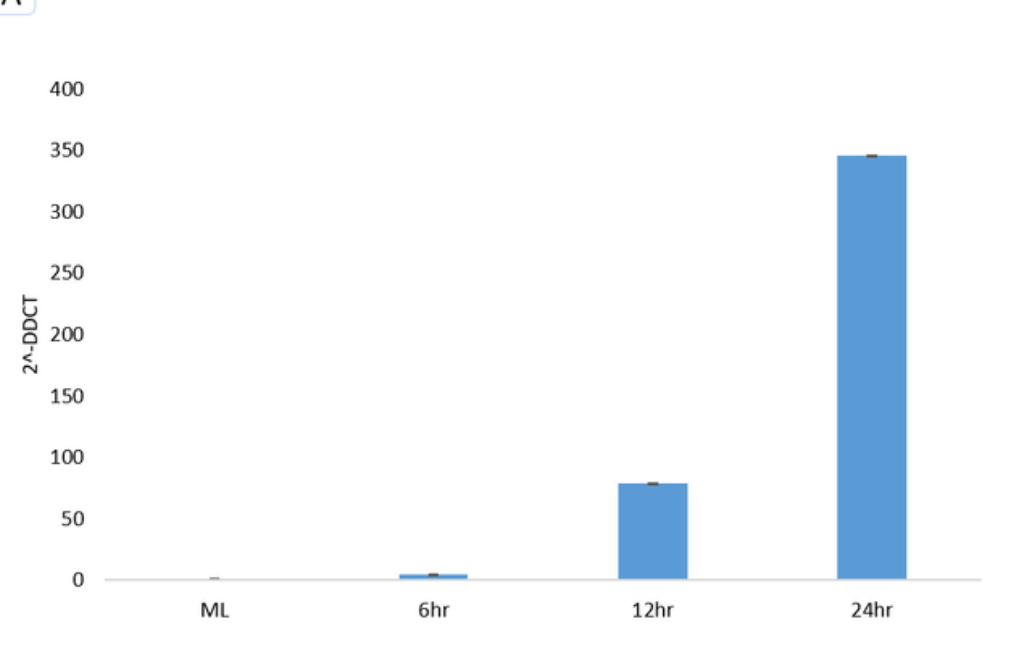

B
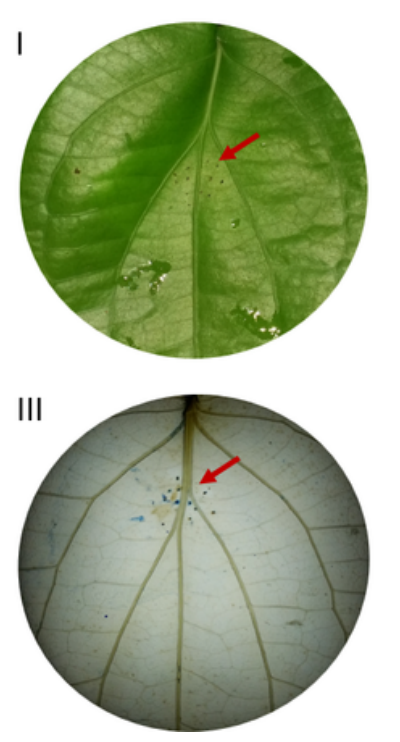
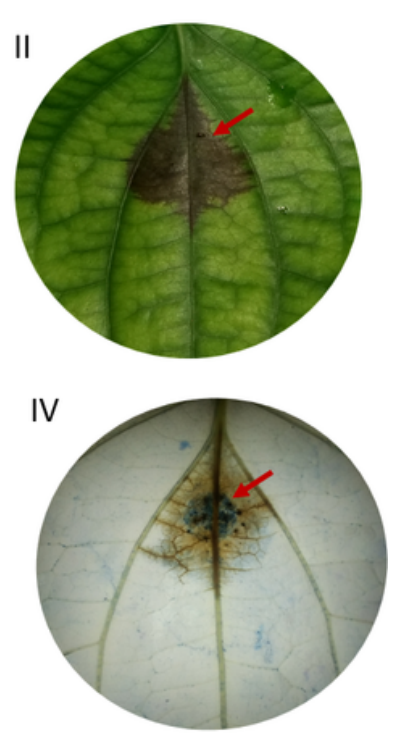

Figure 6

(A)Temporal expression validation of the PnPR-1 gene in Phytophthora capsici infected Piper nigrum plants by RT-qPCR. (B) Mock and Phytophthora capsici infected Piper nigrum leaves after 24 hrs of infection. (I) Mock infected Leaf, (II) Phytophthora capsici infected Piper nigrum leaf, (III) Trypan blue-stained mock infected leaf \& (IV) Trypan blue-stained infected leaf. Red colored arrows indicate the infected regions.

\section{Supplementary Files}

This is a list of supplementary files associated with this preprint. Click to download.

- PRsupplementaryinformation.docx 